\title{
Ecology and conservation of ocelot (Leopardus pardalis) in Northern Quintana Roo, Mexico
}

\author{
Erik Joaquín Torres-Romero ${ }^{1 *}$, Eduardo Espinoza-Medinilla ${ }^{2}$, Marco A. Lazcano-Barrero ${ }^{3}$, Leonardo Maffei ${ }^{4}$ \\ ${ }^{1}$ Colegio de Postgraduados Campus San Luis Potosí, Iturbide 73, Salinas de Hidalgo, CP. 78600, San Luis Potosí. San Luis Potosí, \\ México. Email: ejtr23@hotmail.com (EJTR). \\ ${ }^{2}$ Universidad de Ciencias y Artes de Chiapas. Libramiento Norte Poniente s/n, CP. 29039, Tuxtla Gutiérrez. Chiapas, México. Email: \\ eduardo.espinoza@unicach.mx (EEM). \\ ${ }^{3}$ Reserva Ecológica El Edén A. C, Teocaltiche 207, Fracc. Villas del Sol, CP. 77506, Cancún. Quintana Roo, México. Email: \\ mlazcanobarrero@hotmail.com (MALB). \\ ${ }^{4}$ Wildlife Conservation Society. Av. Roosevelt №6360 Miraflores. Lima, Perú. Email: Imaffei@wcs.org (LM). \\ * Corresponding author
}

Ocelots are at risk of extinction due to anthropogenic pressures. Therefore, it is essential to generate information about this species in zones with climatic and anthropogenic pressures in order to determine appropriate measures for their conservation. Our goals were to determine population density, relative abundance, activity patterns and minimum observed home ranges of ocelots in northern Quintana Roo, Mexico. During 2008, we conducted systematic sampling for 60 days using camera-traps in Northern Quintana Roo, Mexico. The camera-trap grid covered an area of $81 \mathrm{~km}^{2}$ and included El Eden Ecological Reserve. We placed 27 camera-trap stations: 18 with a single camera and 9 with two. Camera-trap stations were spaced approximately 1 kilometer apart. Our total sampling effort was 2160 trap-days. We registered 12 individual ocelots: five males, six females, and one of undetermined sex. The density estimated using CAPTURE program was 14 ind/100 km², while the density estimated for each sex separately was $7 \mathrm{ind} / 100 \mathrm{~km}^{2}$. We estimated a relative abundance index of $1.85 \pm 0.27 \mathrm{in}$ tropical forest, $1.11 \pm 0.22$ in secondary vegetation and $0.185 \pm 0.09$ in savannah. The ocelot was more active at night. The minimum observed home range of male ocelots was larger than that of females, $4.63 \mathrm{~km}^{2}$ and $1.80 \mathrm{~km}^{2}$ respectively. We presented some ecological data of ocelot in El Eden Ecological Reserve and its area of influence. These results constitute an important baseline to make a comparison in areas adjacent that have strong climatic and anthropic pressures. Finally, the area is one of the most important for the conservation of ocelot in México.

El ocelote está en riesgo de extinción debido a factores antropogénicos. Por lo tanto, generar información sobre esta especie en zonas con impactos climáticos y antrópicos se convierte en esencial para determinar medidas adecuadas para su conservación. Nuestros objetivos fueron determinar la densidad de población, abundancia relativa, los patrones de actividad y áreas mínimas de acción del ocelote en el norte de Quintana Roo, México. Durante el 2008 se realizó un muestreo sistemático de 60 días mediante foto-trampeo en el norte de Quintana Roo. El diseño se implementó para muestrear un área de 81 km², que incluye a la Reserva Ecológica El Edén. Se colocaron 27 estaciones de cámaras trampa, 18 fueron sencillas y 9 dobles, cada estación de foto-trampeo tuvo una separación aproximada de 1 kilómetro. En total tuvimos un esfuerzo de muestreo de 2160 días/trampa. Se registraron 12 ocelotes, cinco machos, seis hembras y un individuo sin determinar el sexo. La densidad estimada de ocelotes usando el programa "CAPTURE" fue de $14 \mathrm{ind} / 100 \mathrm{~km}^{2}$, mientras que la densidad estimada para machos $\mathrm{y}$ hembras fue de $7 \mathrm{ind} / 100 \mathrm{~km}^{2}$. El índice de abundancia relativa fue de: $1.85 \pm 0.27$ para selva mediana, $1.11 \pm 0.22$ para vegetación secundaria y $0.185 \pm 0.09$ para sabana. El ocelote presentó su mayor actividad por la noche y el área de acción de los machos fue mayor al de las hembras, $4.63 \mathrm{~km}^{2}$ y $1.80 \mathrm{~km}^{2}$, respectivamente. Se obtuvieron algunos estimadores sobre la ecología del ocelote en la Reserva Ecológica El Edén y su área de influencia. Estos parámetros constituyen una base fundamental para hacer una comparación en áreas adyacentes sujetas a fuertes presiones climáticas y antrópicas. Finalmente, esta zona es una de las de mayor importancia en México para la conservación del ocelote.

Keywords: Activity patterns; camera traps; conservation area; density; endangered species; home range; relative abundance; Yucatan Peninsula

C 2017 Asociación Mexicana de Mastozoología, www.mastozoologiamexicana.org

\section{Introducción}

El ocelote (Leopardus pardalis) es una de las cinco especies de felinos Neotropicales que se distribuyen en México (Aranda 2005). En México, su distribución abarca los estados de Sonora, Sinaloa, Nuevo León, Tamaulipas, Zacatecas, San Luis Potosí, Aguascalientes, Guanajuato, hacia el centro Hidalgo, Puebla, Morelos y Oaxaca y hacia el sur en Chiapas, Quintana Roo hasta Yucatán (Aranda et al. 2014; Bárcenas y Medellín 2010; Galindo-Aguilar et al. 2016; García-Bastida et al. 2016; Hernández-Flores et al. 2013; Iglesias et al. 2008; Martínez-Calderas et al. 2011; Valdez-Jiménez et al. 2013). Este felino al igual que otras especies de vertebrados terrestres, tiene problemas de conservación (e. g., pérdida y fragmentación de hábitat, caza ilegal por depredación de aves de corral y el comercio ilegal de pieles), que en conjunto han provocado una reducción paulatina de su área de distribución en México y en los otros países donde se distribuye, originando un declive poblacional y en otros casos la desaparición local (Aranda 2005; Di Bitetti et al. 2008; Sunquist y Sunquist 2002). Es por ello, que este felino en México se encuentra en la categoría de especie en peligro de extinción y su caza está prohibida (NOM-059, SEMARNAT 2010), mientras que la Unión Internacional para la Conservación de la Naturaleza y los Recursos Naturales (IUCN, por sus siglas en inglés) la clasifica como especie de preocupación menor (IUCN 2015) y su comercialización se encuentra regulada por CITES, ubicándolo en el Apéndice I (CITES 2015). 


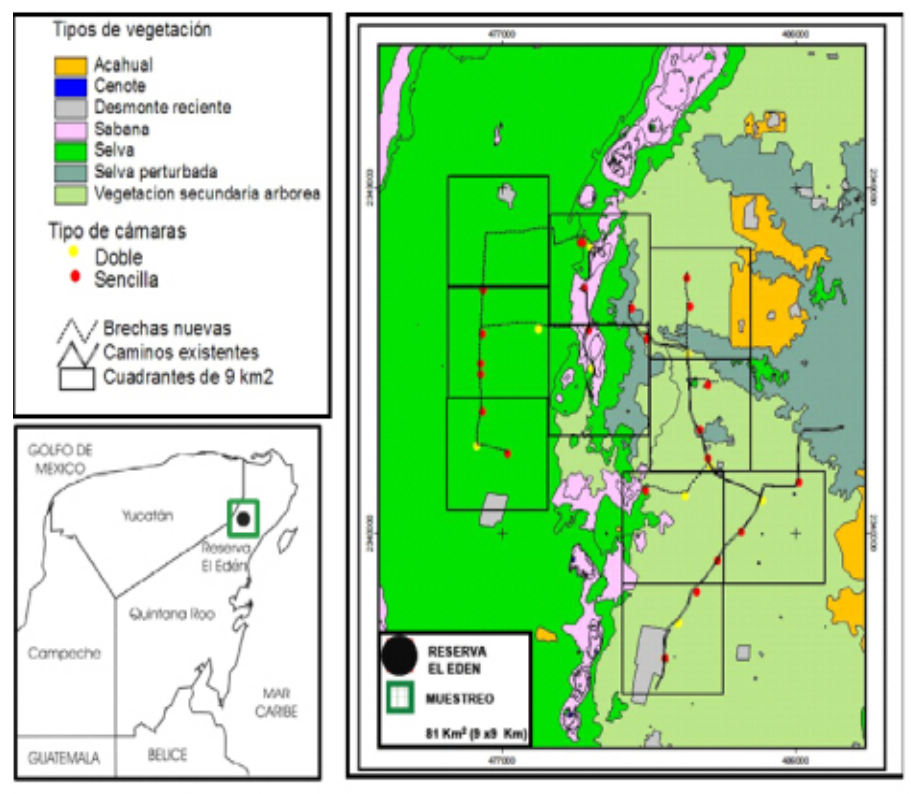

Figura 1. Zona de estudio con el diseño de muestreo propuesto por Chávez et al. (2007) cubriendo un área de $81 \mathrm{Km}^{2}$ y ubicación de las cámaras trampa para el ocelote (Leopardus pardalis) en el norte de Quintana Roo, México.

El norte de Quintana Roo presenta una serie de micro y macro hábitats que le permiten poseer una gran biodiversidad (Lazcano-Barrero et al. 1995). En la península de Yucatán se han registrado por lo menos 212 especies de mamíferos terrestres (Zaragoza-Quintana et al. 2016), y con al menos 404 especies de plantas vasculares (Schultz 2003). El estado de Quintana Roo es una de las áreas de impacto de fenómenos naturales, en la que se han reportado más de 10 huracanes en las últimas dos décadas que han tocado la zona norte y centro de la Península de Yucatán (CONAGUA 2006, 2012), además de que, en los últimos 30 años ha sufrido problemas de incendios, consumiendo más de 100,000 ha de selvas en la zona (CONAFOR, 2016). Asimismo, presenta problemas de intervención humana, entre los cuales se reconocen a la tala ilegal, cacería furtiva, cambio en el uso del suelo y aumento en las actividades turísticas (Lazcano-Barrero et al. 1995; Allen y Rincón 2003). El objetivo de este estudio fue generar información básica sobre la densidad poblacional, abundancia relativa, patrón de actividad y áreas mínimas de acción observadas del ocelote en la región norte de Quintana Roo, misma que ha estado sujeta a perturbaciones climáticas y antrópicas. El generar conocimientos sobre estos aspectos ecológicos pueden constituir un aporte fundamental para los planes de conservación que se realizan en la zona.

\section{Materiales y Métodos}

Área de Estudio. El área se sitúa al norte del estado de Quintana Roo, que incluye a la Reserva Ecológica el Edén (REE). La REE es la primera reserva ecológica privada dedicada a la investigación sobre la conservación y manejo de la biodiversidad en México y está ubicada a los $21^{\circ} 13^{\prime} \mathrm{N},-87^{\circ} 11^{\prime} \mathrm{W}$, en el Municipio de Lázaro Cárdenas (Lazcano-Barrero et al. 1995; Figura 1). La REE, cuenta con una superficie de 3,077 ha, y con al menos cinco tipos de vegetación: a) la selva mediana (vegetación de estructura mediana, semidecidua). b) Los acahuales de diferentes edades, (refiriéndose a vegetación secundaria con diferentes estados de sucesión a consecuencia de diferentes alteraciones antrópicas y climáticas provocados hace más de tres décadas). c) Bosque inundable (tintales y manchones de anonáceas que crecen en sitios inundables). d) Sabanas (que pueden ser áreas abiertas con palmas. e) Vegetación acuática (que crece en zonas pantanosas o cerca de los cenotes; Schultz 2003). Además, la REE pertenece a la región biológica de Yalahau (Gómez-Pompa et al. 2003) y una parte de las selvas tropicales, se encuentra bajo protección federal dentro del Área de Protección de Flora y Fauna Yum-Balam (Navarro et al. 2007). La zona registra una temperatura media anual de $24.7^{\circ} \mathrm{C}$, con una precipitación anual de 1,200 mm (Allen y Rincón 2003).

Diseño de muestreo. Se realizó un muestreo sistemático utilizando trampas cámara digitales de las marcas Cuddeback (expert 3.0 $\mathrm{Mpx}^{\circledR}$ ) y Moultrie (D40 4.0 Mpx ${ }^{\circledR}$ ), ambos modelos contaron con flash visible y siguiendo el diseño del Censo Nacional del Jaguar (CENJAGUAR) propuesto por Chávez et al. (2007). Este consistió en instalar nueve celdas de 3 $x 3 \mathrm{~km}^{2}$ de aproximadamente $9 \mathrm{~km}^{2}$ por celda. Lo que indica que en total se muestreo una área de $81 \mathrm{~km}^{2}$ que incluye a la REE (Figura 1). Cada una de las celdas fue de igual tamaño y no más grande que el área de actividad de un ocelote hembra adulta que varía entre 0.7 y $9.6 \mathrm{~km}^{2}$ (Di Bitetti et al. 2006; Sunquist y Sunquist 2002). En cada celda se colocaron tres estaciones de foto trampeo (dos sencillas y una doble), lo que da un total de cuatro cámaras trampa por celda (Figura 1). Se instalaron un total de 27 estaciones de foto-trampeo, 18 de las cuales fueron estaciones sencillas y nueve dobles (las estaciones dobles permitieron identificar a los individuos por ambos

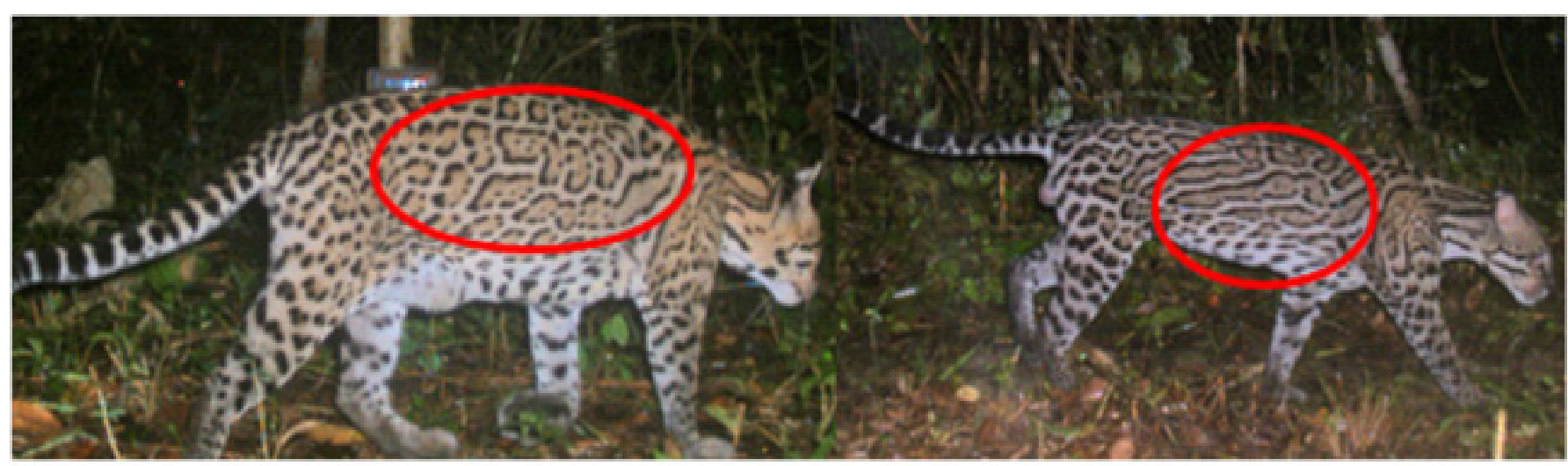

Figura 2. Fotografías del lado derecho de dos ocelotes mostrando el patrón único de marcas en la piel, permitiendo así identificar a distintos individuos. 
lados, importante por el patrón de manchas). Cada estación de foto-trampeo fue separada por lo menos un kilómetro entre estación sencilla o doble. Las cámaras fueron colocadas en diferentes puntos estratégicos para cubrir los tres tipos de vegetación más representativos de la zona: selva mediana, sabana y vegetación secundaria. Las trampas cámara estuvieron activas durante 60 días de foto-registros, iniciando el muestro en la tercera semana del mes de julio y terminando en la segunda semana del mes de septiembre del 2008. La duración total de muestreo no fue superior a 90 días en concordancia con el supuesto de población cerrada (Karanth y Nichols 1998). En total, se obtuvo un esfuerzo de muestreo de 2,160 días/trampa.

La identificación de cada individuo de ocelote (L. pardalis), se estableció por medio del patrón de machas o "marcas únicas" que presenta cada individuo en diferentes partes del cuerpo (Figura 2). Este método permitió diferenciar un individuo de otro, ya que las marcas únicas son patrones naturales de coloración característicos de los felinos manchados (Karanth y Nichols 1998). Se identificaron a los individuos por su sexo (presencia o ausencia de testículos).

Estimación de la abundancia. Para obtener el índice de abundancia relativa (IAR) de los ocelotes, se utilizó la formula sugerida por Maffei et al. (2002) y Sanderson (2004). IAR = (C) EM) x 1,000 días / trampa.

Dónde: $\mathrm{C}$ son las capturas o eventos independientes fotografiados; EM es el esfuerzo de muestreo total (número de cámaras trampa multiplicado por los días de muestreo) y 1,000 días/trampa (frecuencia de captura estandarizando a 1000 días/trampa). Para evitar sobre-estimaciones, se consideraron en los análisis sólo aquellos registros que fueran independientes con un lapso de separación de 60 min entre cada evento. Un registro independiente fue considerado como: 1) registros fotográficos consecutivos en donde no fuera posible identificar al ocelote como un individuo distinto, 2) registros fotográficos consecutivos de individuos diferentes completamente identificables y 3) registros fotográficos con varios individuos considerando a cada individuo como un evento. Posteriormente, se comparó el IAR por tipo de vegetación realizando una prueba

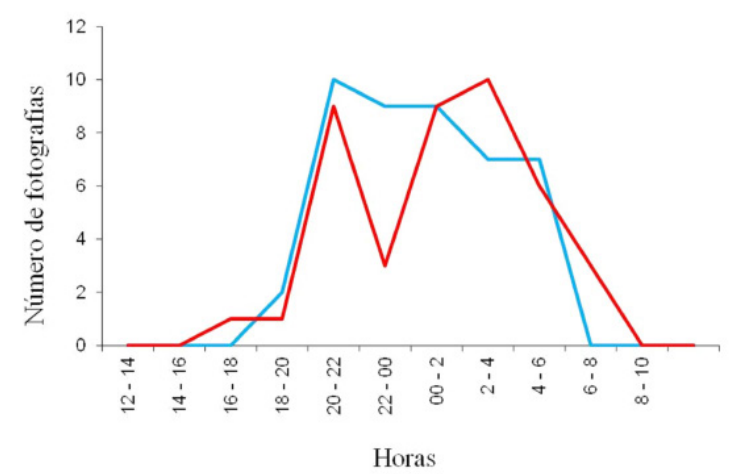

Figura 3. Patrón de actividad del ocelote (machos y hembras) en la zona norte de Quintana Roo, México. Las líneas correspondes en cada caso a ocelotes machos en azul y ocelotes hembras en rojo. de Chi-cuadrada $\left(X^{2}\right)$ y así determinar la preferencia de hábitat (Byers et al. 1984; Krebs 1998), siguiendo la siguiente formula:

$$
\mathrm{X}=\sum_{\mathrm{t}=1}^{\mathrm{K}} \frac{(O-E)^{2}}{\mathrm{E}}
$$

Dónde: $\mathrm{E}=$ frecuencia esperada de registros, $\mathrm{O}=$ frecuencia observada de registros y $\mathrm{K}=$ el número de hábitats comparados.

Patrón de actividad. Para determinar el patrón de actividad de los ocelotes, se utilizó y analizó el horario registrado en todas las fotografías como eventos independientes durante los 60 días de muestreo diferenciando entre machos y hembras. Los registros obtenidos se agruparon por intervalos de dos horas, así sucesivamente para las $24 \mathrm{~h}$. Se consideró la siguiente clasificación para establecer el patrón de actividad: los registros al amanecer se consideraron entre las 06:00 to 8:00 horas, diurno 8:01 to 18:00, crepuscular 18:01 to 20:00, y nocturnos 20:01 to 05:59 horas. Para analizar diferencias en el patrón de actividad del ocelote, se utilizó la prueba de Wald del programa "ACTIVITY" (Rowcliffe 2015).

Estimación de la densidad. Una vez identificados individualmente a los ocelotes, fue posible obtener estimaciones de la densidad poblacional con modelos de captura-recaptura (Silver 2004). Para estimar la densidad de ocelotes, primero se estimaron las abundancias generadas por el programa "CAPTURE" (Karanth y Nichols 1998). Este programa analiza los datos (capturas-recapturas) y determina cuál es el modelo que mejor se ajusta a los datos. Además CAPTURE genera una probabilidad de captura para una población cerrada (p) para hacer estimaciones poblacionales, bajo dos supuestos: 1) la población debe ser cerrada y 2) todos los individuos tienen la misma probabilidad de captura mayor a cero (Silver 2004). Para seleccionar el modelo que mejor se ajusta a los datos, el valor obtenido varía entre 0 a 1, donde el valor más alto indica que el modelo tuvo un mejor ajuste. Posteriormente, la densidad de ocelotes se estimó dividiendo la abundancia relativa generada por CAPTURE entre el área efectiva de muestreo. Esta fue construida a partir del método del polígono

Tabla 1. Registro de frecuencias de ocurrencias de los 12 individuos de ocelote en los diferentes tipos de vegetación.

\begin{tabular}{|c|c|c|c|}
\hline Individuo & Sexo & Fotografías & Tipo de vegetación \\
\hline ocelote A & 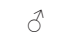 & 13 & vegetación secundaria \\
\hline ocelote B & $\hat{\sigma}$ & 7 & \\
\hline ocelote F & q & 3 & \\
\hline ocelote I & q & 1 & \\
\hline ocelote J & 우 & 1 & \\
\hline ocelote C & 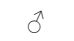 & 12 & selva mediana \\
\hline ocelote D & $\delta$ & 5 & \\
\hline ocelote E & q & 15 & \\
\hline ocelote G & $\hat{0}$ & 1 & \\
\hline ocelote L & q & 7 & \\
\hline ocelote H & 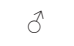 & 3 & sabana \\
\hline ocelote K & $?$ & 1 & \\
\hline
\end{tabular}




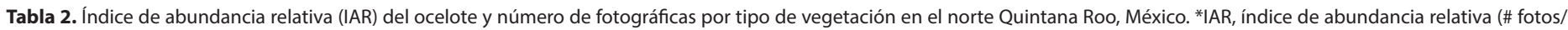
días trampa*1000).

\begin{tabular}{|c|c|c|c|c|}
\hline Individuo & Sexo & $\begin{array}{c}\text { Fotografías } \\
\text { totales }\end{array}$ & $\begin{array}{c}\text { Tipo } \\
\text { de vegetación }\end{array}$ & $\begin{array}{c}\text { IAR* }^{*} \\
\text { y valor de confianza }\end{array}$ \\
\hline ocelote A, B, F, I, J & $\widehat{\jmath}, \hat{\partial}, q, q, q$ & 25 & Vegetación secundaria & $1.111 \pm 0.22$ \\
\hline ocelote $\mathrm{H}, \mathrm{K}$ & $?, \sigma^{\pi}$ & 4 & Sabana & $0.185 \pm 0.09$ \\
\hline ocelote C, D, E, G, L & $\hat{\sigma}, \hat{\sigma}, q, \hat{\sigma}, q$ & 40 & Selva mediana & $1.852 \pm 0.27$ \\
\hline
\end{tabular}

mínimo convexo (Kernohan et al. 2001), que incluyó todas las estaciones de muestreo que estuvieran en los extremos y adicionando a esta, una área de amortiguamiento. El área de amortiguamiento corresponde a la distancia media máxima de desplazamiento (MMDM, por sus siglas en inglés) recorrida por los ocelotes y capturados en dos o más estaciones. El área que cubrió el polígono formada por las cámaras trampa fue de $54 \mathrm{~km}^{2}$, mientras que el área del polígono junto con la zona de amortiguamiento fue de $107.22 \mathrm{~km}^{2}$.

Áreas mínimas de acción. Se estimó el área mínima observada de acción de seis ocelotes (cuatro machos y dos hembras) nombrados como macho M1, M2, M3, M4 y hembras $\mathrm{H} 1$ y $\mathrm{H} 2$ (sólo se consideraron estos ocelotes ya que fueron registrados en más de dos estaciones). Posteriormente, el área mínima observada de acción se estimó con el método del polígono mínimo convexo (Kernohan et al. 2001). Uniendo los puntos extremos donde los ocelotes fueron registrados. Se realizó una prueba de bondad de ajuste Chi-cuadrada $\left(X^{2}\right)$ para evaluar diferencias en el área de acción entre machos y hembras.

\section{Resultados}

Identificación de ocelotes. Se obtuvieron 90 fotografías de ocelotes, de las cuales 68 fueron los registros fotográficos que se analizaron para su identificación. En total, se identificaron a 12 individuos adultos, cinco machos, seis hembras y un individuo al que no fue posible determinar el sexo, en donde cada ocelote fue nombrado en forma alfabética (A-L; Tabla 1). De los registros obtenidos, la frecuencia de ocurrencia indicó que la selva mediana obtuvo mayores registros fotográficos en comparación la vegetación secundaria y sabana (Tabla 1 y 2).

Estimación de Abundancias Relativas. El índice de abundancia relativa (IAR) fue estimado en los tres tipos de vegetación, encontrándose el mayor IAR para la selva mediana de $1.85 \pm$ 0.27 , mientras que la vegetación secundaria fue de $1.11 \pm 0.22$

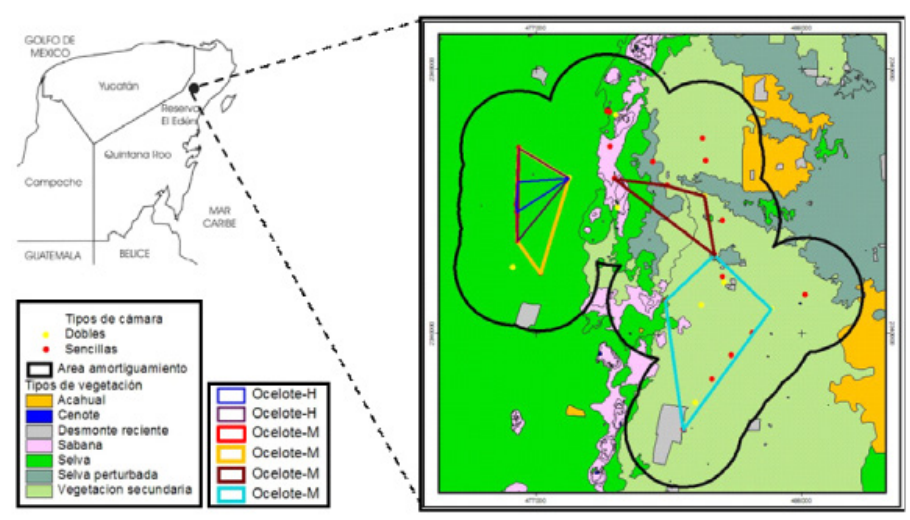

Figura 4. Ocelotes machos y hembras utilizados para crear el área de acción para la zona de muestreo en el norte de Quintana Roo, México. y finalmente, el menor IAR fue para la sabana, de $0.185 \pm 0.09$. Además, la proporción de ocelotes hembras fue mayor para la vegetación secundaria, mientras que para la selva mediana y sabana la proporción de ocelotes hembras fue menor (Tabla 2).

Patrón de actividad. En relación al número de fotografías registradas, los ocelotes machos y hembras mostraron un pico de mayor actividad por la noche, para los machos fue alrededor de las 20:00 y 22:00 h., mientras que para las hembras se registró entre las 2:00 y 4:00 $\mathrm{h}$. Las hembras presentaron dos picos en su patrón de actividad, ambos registrados por la noche. El primer pico fue de inactividad registrado entre las 22:00 y 00:00 h. El segundo, se registró regresando a su actividad entre las 00:00 y 2:00 h. Las hembras y los machos presentaron un periodo de inactividad registrado en el día. Para las hembras se registró entre las 10:00 y 16:00 h., mientras que para los machos se registró entre las 8:00 y las 18:00 h. La comparación del patrón de actividad entre períodos mostró que los ocelotes fueron significativamente más activos por la noche en comparación con los demás periodos del día ( $\mathrm{W}=3.74 ; P<0.05)$.

Estimación de densidades. La densidad estimada para ocelotes sin diferenciar sexo fue de $14 \mathrm{ind} / 100 \mathrm{~km}^{2}$, mientras que, la estimación para machos y hembras fue de 7 ind $/ 100 \mathrm{~km}^{2}$. De acuerdo con los resultados, el modelo de heterogeneidad $M(h)$ con un valor de 1.00, fue el modelo que mejor se ajustó a los datos para evaluar la densidad de ocelotes en la zona de estudio (Tabla 3). Con la densidad obtenida de $14 \mathrm{ind} / 100$ $\mathrm{km}^{2}$, se estima cautelosamente que probablemente podrían existir unos 560 ocelotes para las 400,000 ha de selvas que corresponden a las Áreas de Conservación en la Península de Yucatán (ACPY), que incluyen al Parque Natural Reserva Ría Lagartos, Área de Protección de Flora y Fauna Yum Balam, Área Natural Protegida "El Zapotal", la Reserva Ecológica el Edén, Reserva estatal Bocas de Dzilam, y región de Yalahau (Tabla 4).

Área mínima de acción. El área mínima observada de acción de los ocelotes machos fue de $4.63 \mathrm{~km}^{2}$ y para hembras fue de $1.80 \mathrm{~km}^{2}$ (Tabla 5). Al comparar los valores se encontró que entre machos y hembras existen diferencias estadísticamente significativas $\left(\mathrm{X}^{2}=259.47 ; \mathrm{g}\right.$. I. $\left.=1 ; P<0.05\right)$. El área de acción de machos es 2.57 veces mayor al de las hembras, con una sobreposición entre individuos del mismo y de distintos sexos (Figura 4).

\section{Discusión}

Preferencia de hábitat, patrón de actividad y área mínima de acción. Los ocelotes se pueden encontrar en diversos tipos de hábitats, que van desde bosques húmedos, sabanas, bosques deciduos, zonas con matorral, zonas perturbadas, selvas altas y medianas, además de que, se han registrado en diferentes altitudes desde los 0 a los 3,800 msnm (Maffei et al. 2005; Mar- 


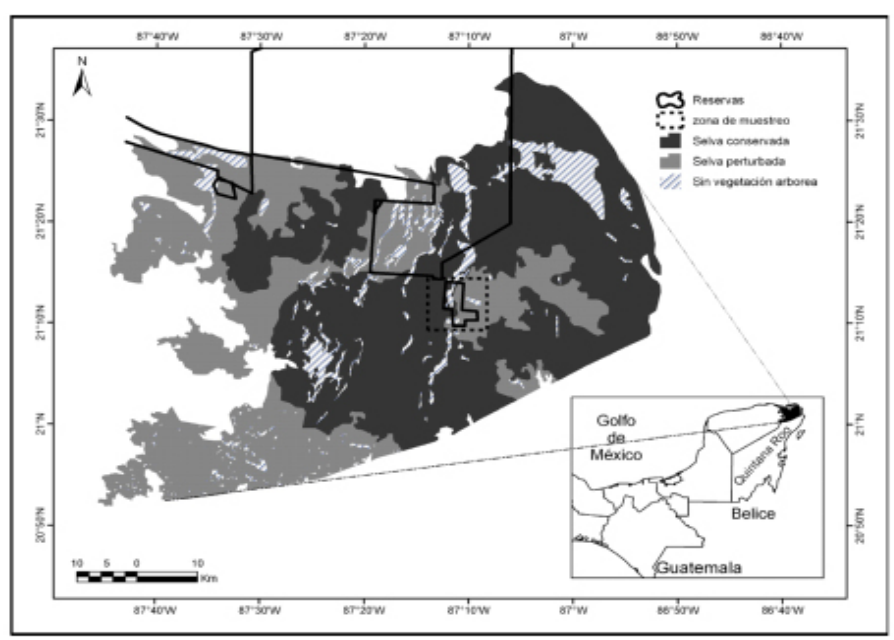

Figura 5. Áreas Naturales Protegidas de carácter privadas y nacionales, utilizadas para hacer una estimación cautelosa de la población del ocelote (Leopardus pardalis) en la zona norte de la Península de Yucatán.

tínez-Calderas et al. 2011; Valdez-Jiménez et al. 2013; Aranda et al. 2014; Pérez-Irineo y Santos-Moreno 2014). Sin embargo, Sunquist y Sunquist (2002), Emmons (1988) y Emmons et al. (1989), sugieren que los ocelotes prefieren las coberturas vegetales densas, ya que estas les proporciona hábitats de refugio ante posibles depredadores y del mismo hombre, además de sitios de descanso y de seguridad para proteger a sus crías, así como de lugares para la búsqueda de alimento. Jackson et al. (2005), realizaron un trabajo por medio de telemetría en Texas y comprobaron que los ocelotes prefieren aquellos parches de vegetación con coberturas densas.

En el presente estudio, los ocelotes prefirieron aquellos hábitats más conservados, como es el caso de la selva mediana subperennifolia, visitando menos los sitios abiertos tales como la vegetación secundaria y la sabana. Esto sugiere la importancia que tiene la selva mediana subperennifolia, ya que ésta, se encuentra con menor grado de perturbación humana (e.g., cambio del uso del suelo, incendios y tala ilegal) y por ende posee una cobertura vegetal conservada que pudiera proporcionar lugares seguros para su resguardo, alimentación y hábitats de paso, principalmente. En este sentido, se ha documentado que uno de los principales problemas de conservación del ocelote es la destrucción y pérdida paulatina de las áreas con cobertura vegetal densa (Emmons et al. 1989; Laack 1991).
Es por ello que una estrategia de conservación debe incluir la protección de áreas con cobertura vegetal lo más densa posible dentro y fuera de áreas naturales protegidas para garantizar que estos felinos puedan tener hábitats disponibles para sobrevivir a medio y lanzo en la región y en México.

En relación al periodo de actividad, se reporta que pueden estar activos durante todo el día, con una mayor actividad por las noches (Di Bitetti et al. 2006; Maffei et al. 2005, Pérez-Irineo y Santos-Moreno 2014), además de que, su actividad también depende de la actividad de sus presas potenciales (e. g., roedores, reptiles y pequeños mamíferos y aves; Murray y Gardner 1997). Nuestros registros de foto captura sugieren que los ocelotes presentan un periodo de actividad tanto de día como de noche, pero que son preferentemente nocturnos.

Las estimaciones de las áreas de acción de los ocelotes muestran que estas pueden variar entre $3.5 \mathrm{~km}^{2}$ y $17.7 \mathrm{~km}^{2}$ para los machos y entre $0.7 \mathrm{~km}^{2}$ y $14.6 \mathrm{~km}^{2}$ para las hembras (Emmons et al. 1989). Navarro (1985), reporta que los machos presentan una área de acción de $2.52 \mathrm{~km}^{2}$ y para las hembras de $2.07 \mathrm{~km}^{2}$, mientras que el estudio realizado por Martínez-Meyer (1997), sugiere que las hembras tienen un área de acción superior a la de los machos con $5.68 \mathrm{~km}^{2}$ y $5.23 \mathrm{~km}^{2}$, respectivamente. Se reporta, que los machos suelen tener un área de acción más amplia con respecto a las hembras, lo cual podría deberse por una lado a que los machos tienen que defender su territorio y por el otro para la búsqueda de hembras para poder reproducirse (Murray y Gardner 1997; Sunquist 1991). Además, existe evidencia en la que se sugiere que el área de acción de los ocelotes puede ser compartida por individuos de ambos sexos, es decir la sobreposición se puede presentar entre hembras que tengan afiliación por la línea materna (Murray y Gardner 1997).

Aquí reportamos con base a nuestros registros que el área mínima de acción observada del ocelote en el norte de Quintana Roo se encuentra con valores promedio de los resultados reportados en otras áreas y tipos de hábitats (Di Bitetti et al. 2006; Emmons et al. 1989; Laack 1991; Navarro 1985; Maffei et al. 2005; Martínez-Meyer 1997; Salom 2005; Tabla 5). Además, encontramos que existe una sobreposición de las áreas mínimas observadas de acción entre ocelotes machos y hembras, no encontrando el patrón entre ocelotes machos, lo que podría

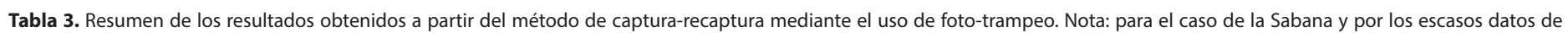
captura-recaptura, no fue posible hacer una estimación.

\begin{tabular}{|c|c|c|c|c|c|c|c|}
\hline Categorías & PCE & PC & DE & MMDM (m) & Amt $\left(\mathbf{k m}^{2}\right)$ & M & $\mathbf{P}$ \\
\hline Machos & 0.066 & $7 \pm 1.19$ & 7 & 4,510 & 39.33 & $M(h)$ & $\mathrm{z}=-0.883 ; P=0.188$ \\
\hline Hembras & 0.073 & $7 \pm 2.12$ & 7 & 2,720 & 20.56 & $M(h)$ & $z=0.150 ; P=0.559$ \\
\hline \multicolumn{8}{|l|}{ Tipo de vegetación } \\
\hline Selva Mediana & 0.100 & $6 \pm 1.63$ & 23 & 3,285 & 26.08 & $M(h)$ & $z=0.534 ; P=0.703$ \\
\hline Vegetación secundaria & 0.043 & $7 \pm 2.12$ & 8 & 3,886 & 63.47 & $M(h)$ & $\mathrm{z}=-0.816 ; P=0.207$ \\
\hline \multicolumn{8}{|l|}{ Sabana } \\
\hline TOTAL & 0.053 & $15 \pm 2.59$ & 14 & 3,542 & 107.22 & $M(h)$ & $\mathrm{z}=-0.383 ; P=0.350$ \\
\hline
\end{tabular}

PCE $=$ Probabilidad de captura estimada $; \mathrm{PC}=$ Población estimada con CAPTURE $; \mathrm{DE}=$ Densidad estimada (individuos $\left./ 100 \mathrm{~km}{ }^{2}\right) ; \mathrm{MMDM}=$ Promedio de las distancias máximas de desplazamiento; $\mathrm{Amt}=$ Área de amortiguamiento; $\mathrm{M}=$ Modelo; $\mathrm{M}(\mathrm{h})=$ Modelo de JACKNIFE, probabilidad de captura heterogénea; $\mathrm{P}=$ Prueba de población cerrada 
Tabla 4. Estimación del tamaño poblacional de ocelotes (sin diferenciar sexo 14 ind $/ 100 \mathrm{~km}^{2}(\mathrm{DA})$, de machos y hembras $\left.7 \mathrm{ind} / 100 \mathrm{~km}^{2}(\mathrm{DB})\right)$ para las áreas de conservación privadas y nacionales al norte de la Península de Yucatán.

\begin{tabular}{lccc}
\hline \multicolumn{1}{c}{ Áreas de Conservación } & Sup. km² & DA & DB \\
\hline Áreas de conservación en la Península de Yucatán (ACPY) & 1,400 & 196 & 98 \\
Región Yalahau y Ría Lagartos (incluyendo ACPY) & 4,000 & 560 & 280 \\
\hline
\end{tabular}

ACPY: Parque Natural Reserva Ría Lagartos, Área de Protección de Flora y Fauna Yum Balam, Área Natural Protegida “El Zapotal”, la Reserva Ecológica el Edén, Reserva estatal Bocas de Dzilam, y región de Yalahau, superficie en $\mathrm{km}^{2}$ tomado y modificado de Faller et al. 2006

coincidir parcialmente con lo reportado por Murray y Gardner (1997) y Sunquist (1991). Por tanto, y con base a lo sugerido por estos autores, cabe la posibilidad de considerar que las hembras estuvieran en período reproductivo, o del cuidado de sus crías, ya que se encontró una sobreposición del área mínima de acción entre hembras y machos. También, encontramos que los ocelotes machos no presentaron una sobreposición del área mínima de acción (Figura 4). Cabe mencionar que, cuatro de las seis estimaciones del área mínima de acción de los ocelotes se registraron en la selva mediana. Estas diferencias pueden estar relacionadas a la preferencia del tipo de hábitat, ya que los movimientos de los ocelotes tienden a concentrarse en aquellas zonas que les proporcionen más oportunidades de recursos alimenticios y de refugio (Martínez-Meyer 1997).

Estimación poblacional. La densidad poblacional de ocelotes estimada sin diferenciar el sexo fue de $14 \mathrm{ind} / 100 \mathrm{~km}^{2}$. Esta densidad estimada es similar con lo registrado en otros sitios como Argentina y Guatemala (Di Bitetti et al. 2006; Moreira et al. 2007), pero diferente a lo registrado en Costa Rica, Bolivia, Texas, Belice y Los Chimalapas en México (Dillon y Kelly 2007, 2008; Maffei et al. 2005; Salom 2005; Haines et al. 2006; Pérez-Irineo y Santos-Moreno 2014), ya que en estos lugares presentan densidades superiores. Estas diferencias en las densidades estimadas podrían variar en el espacio y tiempo, además de estar relacionadas a factores metodológicos, tales como: número de estaciones de muestreo (e. g., sencillas o dobles), días/trampa, distancia entre estaciones de trampeo, y temporada de muestreo (e. g., lluvias o secas). Aunado a esto, las densidades estimadas en cada región podrían estar determinadas por disturbios climáticos y antrópicos (e. g. incendios, huracanes), tipo de hábitat (e. g., bosques de pino, bosques tropicales, selvas perennifolias, matorrales), por características del paisaje (e. g., áreas con pérdida y modificación de sus

Tabla 5. Estimación del área de acción de ocelotes en diferentes sitios y del presente estudio.

\begin{tabular}{lccll}
\hline \multicolumn{1}{c}{ Lugar } & \multicolumn{2}{c}{ Sexo $\left(\mathbf{k m}^{\mathbf{2}}\right)$} & Método & \multicolumn{1}{c}{ Autor } \\
& Machos & hembras & & \\
\hline Perú & 5.9 & 1.6 & Telemetría & Emmons et al. 1989 \\
Texas, EE. UU. & 6.25 & 2.87 & Telemetría & Laack 1991 \\
Texas, EE. UU. & 2.52 & 2.07 & Telemetría & Navarro 1985 \\
Jalisco, México & 5.23 & 5.68 & Telemetría & Martínez-Meyer 1997 \\
Costa Rica & 6.19 & & Trampas cámara & Salom 2005 \\
Argentina & 13.41 & 6.01 & Telemetría & Di Bitetti et al. 2006 \\
Bolivia & 3.1 & 2.45 & Telemetría & Maffei et al. 2005 \\
Quintana Roo & 4.63 & 1.8 & Trampas cámaras & Presente estudio \\
\hline
\end{tabular}

coberturas forestales y áreas con algún grado de protección), y por disponibilidad de recursos alimenticios, ocasionando que los estudios realizados en otras áreas y tipos de hábitats no sean comparables (Dillon y Kelly 2007, 2008).

Ávila-Nájera et al. (2015), realizaron un estudio en nuestra misma zona de estudio durante los años 2008, 2010, 2011, y 2012 , en la que reportan una densidad de 13.86 ind/100 $\mathrm{km}^{2}$ para el 2010 y para el mismo periodo de muestro del presentado en nuestro estudio. Sin embargo, para los años posteriores reportan una densidad por debajo de la reportada para el 2010. Esto probablemente se deba a aspectos tales como: i) diferentes periodos de muestreo, ii) presencia de alteraciones antrópicas y climáticas (e. g., incendios forestales y huracanes), que promueven la dispersión de las especies, y iii) movilidad de los ocelotes hacia otros hábitats para la búsqueda de alimento, reproducción y cuidado de crías. Por tanto, se infiere que estos factores, pudieran afectar la dinámica de la especie dentro del mismo espacio y tiempo provocando una cierta reacción en el cambio del patrón de movimiento ante los efectos antrópicos y climáticos.

Áreas de conservación. En la zona norte de la Península de Yucatán existen aproximadamente unas 400,000 ha de selvas protegidas para su conservación (Faller et al. 2006), y que han estado sujetas a alteraciones antrópicas moderadas a severas (e. g., apertura de diversas carreteras, tales como la autopista Cancún-Mérida e Ideal-Cancún). Esta perturbación puede provocar que la zona se encuentre con problemas de conectividad con las selvas del sur de la Península (e. g., Calakmul, Balam ka`ax, Balam ku, Balam kim y Sian Kaán). Esta falta de conectividad podría inducir a un aislamiento poblacional de ocelotes y otros grupos de vertebrados terrestres, y que, a mediano y largo plazo pueda reflejarse en problemas de endogamia, enfermedades y en otros casos la extirpación local.

Considerando que la estimación obtenida de ocelotes fue de $14 \mathrm{ind} / 100 \mathrm{~km}^{2}$, es posible proporcionar un dato del posible tamaño poblacional del ocelote en el norte de la Península de Yucatán. De tal manera que se podría hacer una estimación con cautela de una población de ocelotes, en la que podrían existir 560 ocelotes en las 400,000 ha de selvas que cubren las Áreas Naturales Protegidas (Figura 5, Tabla 4). Sin embargo, consideramos la importancia de realizar estudios más profundos en el tiempo y espacio en áreas adyacentes con hábitats similares a los estudiados para obtener estimaciones más sólidas y robustas sobre la densidad poblacional de ocelotes en la zona norte de la Península.

A modo de conclusión, el presente estudio brinda información indispensable sobre algunos aspectos de la ecología del ocelote, entre esta, encontramos que los ocelotes presentan un patrón de actividad nocturno y su actividad empieza a disminuir en el amanecer, donde las hembras permanecen activas más horas que los machos. El índice de abundancia relativa indica que los ocelotes son más abundantes en la selva mediana seguido de vegetación secundaria y por último la sabana. Nuestros resultados muestran que existe una sobreposición de las áreas mínimas de acción observada de los ocelotes entre individuos de sexos diferentes, en donde, 
Tabla 6. Estimación de la densidad de ocelotes para diferentes sitios de muestreo y del presente estudio. Dias de muestreo (DM), Densidad (ind $/ 100 \mathrm{~km}^{2}$; Den), Estaciones de trampeo (ET).

\begin{tabular}{lcccl}
\hline \multicolumn{1}{c}{ Sitio de Estudio } & DM & Den & ET & \multicolumn{1}{c}{ Autor } \\
\hline Bolivia & 60 & $30-56$ & & Maffei et al. 2005 \\
Costa Rica & 90 & 23.57 & 12 & Salom 2005 \\
Texas & $60-76$ & 30.00 & & Haines et al. 2006 \\
Argentina & 90 & 13.36 & 36 & Di Bitetti et al. 2006 \\
Belice & & 22.85 & 19 & Dillon y Kelly 2007 \\
Guatemala & 21 & 14.70 & 21 & Moreira et al. 2007 \\
Belice & $30-90$ & 25.88 & 17 & Dillon y Kelly 2008 \\
Argentina & 96 & 17.60 & 42 & Di Bitetti et al. 2008 \\
Chimalapas, México & & $22-38$ & 29 & Pérez-Irineo y Santos-Moreno 2014 \\
N. Quintana Roo & $65-82$ & $1.4-13.8$ & $22-27$ & Ávila-Nájera et al. 2015 \\
N. Quintana Roo & 60 & 14.00 & 27 & Presente estudio \\
\hline
\end{tabular}

el tamaño promedio del área mínima de acción es mayor para los machos. Finalmente, este trabajo representa un avance relevante sobre la situación del ocelote (L. pardalis) en la REE y su área de influencia, constituyendo una línea base fundamental para hacer una comparación en áreas adyacentes que están sujetas a fuertes presiones climáticas y antrópicas.

\section{Agradecimientos}

Agradecemos a C. MacSwiney y a dos revisores anónimos por sus comentarios constructivos al manuscrito. Agradecer a A. Noss por los aportes hechos al trabajo. A la Reserva Ecológica el Edén y al personal de la misma por brindar el apoyo logístico, administrativo y de campo. Agradecemos la valiosa colaboración en la recopilación de datos a J. Castillo y F. Arenas. Este estudio se realizó como parte del Censo Nacional del Jaguar y sus presas financiado por la CONANP, la UNAM y La Alianza WWF Telcel. Financiamiento adicional para el desarrollo del trabajo de campo fue otorgado por el Sr. Bush y F. Escobar de la empresa Halach Winick, a quienes agradecemos su valioso apoyo. El primer autor agradece al Consejo de Ciencia y Tecnología de México (CONACYT) por la beca otorgada durante sus estudios de maestría.

\section{Literatura citada}

AlLEN, M. F, y E. Rincón. 2003. The Changing Global Environment and the Lowland Maya: Past Patters and Current Dynamics. Pp. 13-29 in The Lowland Maya Area: three millennia at the humanwildland interface. The Haworth Press. New York, EE. UU.

Aranda, M., F. Botello, E. Martínez-Meyer, y A. Pineda. 2014. Primer registro de ocelote (Leopardus pardalis) en el Parque Nacional Lagunas de Zempoala, Estado de México y Morelos, México. Revista Mexicana de Biodiversidad 85:1300-1302.

Aranda, M. 2005. Ocelote. Pp. 359-361, en Los mamíferos silvestres de México (Ceballos, G., y G. Oliva, eds.). CONABIO, Fondo de Cultura Económica. Ciudad de México, México.

Ávila-Nájera, D. M., C. Chávez, M. A. Lazcano-Barrero, S. PérezElzalde, Y J. L. Alcántara-Carbajal. 2015. Estimación poblacional y conservación de felinos (Carnivora: Felidae) en el norte de Quintana Roo, México. Revista de Biología Tropical 63:799-813.
Bárcenas, H., y R. A. Medellín. 2010. Ocelot (Leopardus pardalis) in Aguascalientes, Mexico. Southwestern Naturalist 55:447449.

Byers, R. C., R. K. Steinhorst, y P. R. Krausman. 1984. Clarification of a technique for analysis of utilization-availability data. Journal of Wildlife Management 48:1050-1053.

Chavez, C., G. Ceballos, R. Medellín, y H. Zarza. 2007. Primer censo nacional del jaguar. Pp. 133-141 in Conservación y Manejo del Jaguar en México estudios de caso y perspectivas. CONABIOUNAM-Alianza WWF, Telcel. Ciudad de México, México.

COMIIIÓN NACIONAL FORESTAL (CONAFOR). 2016. Incendios Forestales Generalidades, México. Disponible en: http://www.conafor. gob.mx/programas nacionales forestales/incendios/

Convención Sobre el Comercio Internacional de Especies Amenazadas de Fauna y Flora Silvestres (CITES). 2015. Convención sobre el Comercio Internacional de Especies Amenazadas de Fauna y Flora Silvestres (CITES). Apéndices I, II y III en vigor a partir del 24 de julio de 2010. http://www.cites.org/. Última visita: 30 marzo 2015.

Comisión Nacional del Agua (conagua). 2006. Análisis de las temporadas de huracanes de los años 2004 y 2005 en México, Comisión Nacional del Agua. Ciudad de México, México.

Comisión Nacional del Agua (conagua). 2012. Análisis de las temporadas de huracanes de los años 2009, 2010 y 2011 en México, Comisión Nacional del Agua. Ciudad de México, México.

Di Bitetti, M. S., A. Paviolo, C. De Angelo, y Y. Di Blanco. 2008. Local and continental correlates of the abundance of a neotropical cat, the ocelot (Leopardus pardalis). Journal of Tropical Ecology 24:1-12.

Di Bitetti, M. S., A. Paviolo, y C. De Angelo. 2006. Density, habitat use and activity patterns of ocelots (Leopardus pardalis) in the Atlantic Forest of Misiones, Argentina. Journal of Zoology 270:153-163.

DILLON, A., Y M. KelLy. 2007. Ocelot Leopardus pardalis in Belize: the impact of trap spacing and distance moved on density estimates. Oryx 41:469-477.

Dillon, A., y M. Kelly. 2008. Ocelot home range, overlap and density: comparing radio telemetry with camera trapping. Journal of Zoology 275:391-398.

Emmons, L. H., P. Sherman, D. Bolster, A. Goldizen, y J. Terborgh. 1989. Ocelot behavior in moonlight. Pp. 233-242 in Advances in Neotropical Mammalogy. Sandhill Crane Press. Gainesville, EE.UU. Emmons, L. H. 1988. A field study of ocelots (Felis pardalis) in Peru. Reviewd'Ecologie (Terre Vie) 43:133-157.

Faller, J. C., C. Chávez, S. Johnson, y G. Ceballos. 2006. Estimación de una población de jaguar en el norte de la Península de Yucatán. Pp. 259 in Conservación y manejo del jaguar en México: estudios de caso y perspectivas. Conabio-Alianza WWF. Telcel-UNAM. Ciudad de México, México.

Galindo-Aguilar, R. E., L. Cacelin-Castillo, O. C. Rosas-Rosas, M. G. Bravo-Vinaja, J. L. Alcantara-Carbajal, y V. Vazquez-García. 2016. First records of ocelot in tropical forests of the Sierra Negra of Puebla and Sierra Mazateca de Oaxaca, Mexico. Therya 7:205-211.

García-Bastida, M., F. Martínez-De La Fuente, A. Vázquez-Venegas, y J. L. Peña-MondRagón. 2016. A new record of ocelot in Parque Ecológico. Therya 7:1-6. 
Gómez-Pompa, A., M. F. Allen, S. Fedick, y J. J. Jiménez-Osorio. 2003. The Lowland Maya Area. Three Millennia at the HumanWildland Interface Binghamton. The Haworth Press. New York, EE. UU.

Haines, A., J. Janecka, M. Tewes, L. Grassman, y P. Morton. 2006. The importance of private lands for ocelots (Leopardus pardalis) conservation in the United States. Oryx 40:1-5.

Hernández-Flores, S. D., G. VARgas-Licona, y G. SÁnchez-Rojas. 2013. First records of the Ocelot (Leopardus pardalis) in the state of Hidalgo, Mexico. Therya 4:99-102.

Iglesias, J., V. Sánchez-Cordero, G. Magaña-Cota, R. Bolaños, M. Aranda, R. Hernández, y F. Botello. 2008. Noteworthy records of margay, Leopardus wiedii and ocelot, Leopardus pardalis in the state of Guanajuato, Mexico. Mammalia 72:347-349.

International Union For Conservation of Nature (UICN). 2015. The IUCN red list of threatened species. Version 2014.3. http:// www.iucnredlist.org. Última visita: 6 enero 2015.

JACKSON, V. L., L. L. LAACK, y E. G. Zimmerman. 2005. Landscape metrics associated with hábitat use by ocelots on south Texas. Journal of Wildlife Management. 69:733-738.

KaRANTH, K. U, y J. D. Nichols. 1998. Estimation of tiger densities in India using photographic captures and recaptures. Ecology 79:2852-2862.

Kernohan, B. J., R. A. Gitzen, y J. J. Millspaugh. 2001. Analysis of animal space use and movement. Pp. 125-166 en Radio Tracking and Animal Populations. Academic Press. San Diego, EE. UU.

KreBS, C. H. 1998. Ecological Methodology. $2^{\text {da }}$. Ed. Harper y Row Publishers. New York, EE. UU.

LAACK, L. L. 1991. Ecology of the ocelot (Felis pardalis) in south Texas. Thesis of master. Texas University. Kingsville, EE. UU.

Lazcano-Barrero, M. A., M. Vázquez-Sánchez, I. March, H. Núñez, y M. Fuller. 1995. La región de Yalahau: propuesta para el establecimiento de una zona de conservación y desarrollo sostenible en el norte de Quintana Roo, CECRN, Colegio de la Frontera Sur. Chetumal, México.

Maffel, L., E. Cúellar, y A. Noss. 2002. Uso de trampas cámara para la evaluación de mamíferos en el ecotono ChacoChiquitanía. Revista Boliviana de Ecología y Conservación Ambiental 11:55-65.

Maffel, L., A. J. Noss, E. Cuéllar, y D. I. Rumiz. 2005. Ocelot (Felis pardalis) population densities, activity, and ranging behaviour in the dry forests of eastern Bolivia: data from camera trapping. Journal of Tropical Ecology 21:349-353.

Martínez-Meyer, E. 1997. Estudio ecológico del ocelote (Leopardus pardalis) en la zona de Chamela, Jalisco, México. UNAM, Tesis de Maestría. Ciudad de México, México.

Martínez-Calderas, J. M., O. C. Rosas-Rosas, J. F. Martínez-Montoya, L. A. Tarango-Arámbula, F. Clemente-Sánchez, M. M. Crosby-Galván, y M. D. SÁnchez-Hermosillo. 2011. Distribución del ocelote (Leopardus pardalis) en San Luis Potosí, México. Revista Mexicana de Biodiversidad 82:997-1004.

Moreira, J., R. García, R. Mcnab, T. Dubón, F. Córdova, y M. Córdova. 2007. Densidad de ocelotes (Carnivora: Leopardus pardalis) en la parte este del Parque Nacional Mirador Río Azul, Guatemala. WCS-Guatemala. Petén, Guatemala.

MuRRAY, J. L., y G. L. GARDNER. 1997. Leopardus pardalis. Mammalian Species 548:1-10.
Navarro, D. 1985. Status and distribution of the ocelot (Felis pardalis) in South Texas. Tesis de Maestría. Texas University. Kingsville, EE. UU.

Navarro, C. J., J. F. Remolina, y J. J. Pérez. 2007. El jaguar en Yum Balam y el Norte de Quintana Roo. Pp. 123-132 in Conservación y manejo del jaguar en México: estudio de caso y perspectivas. CONABIO, Alianza WWF, Telcel, Universidad Nacional Autónoma de México. Ciudad de México, México.

Pérez-Irineo, G., y A. Santos-Moreno. 2014. Density, distribution, and activity of the ocelot Leopardus pardalis (Carnivora: Felidae) in Southeast Mexican rainforests. Revista de Biología Tropical 62:1421-1432.

RowCLIFFE, M. 2015. Package "activity" Animal Activity Statistics. https://cran.r-project. org/web/packages/activity/index.html SAlom, R. 2005. Ecología del jaguar (Panthera onca) y el Manigordo (Leopardus pardalis) carnivora: felidae, en el Parque Nacional Corcovado, Costa Rica. Tesis de Maestría. Universidad de Costa Rica. San José, Costa Rica.

Semarnat (Secretaria del Medio Ambiente y Recursos Naturales). 2010. Norma Oficial Mexicana NOM-059-ECOL-2010, Protección ambiental-Especies nativas de México de flora y fauna silvestres-Categorías de riesgo y especificaciones para su inclusión, exclusión o cambio-Lista de especies en riesgo. Ciudad de México, México.

Sanderson, J. G. 2004. Protocolo para Monitoreo con Cámaras para Trampeo Fotográfico. Tropical Ecology Assessment and Monitoring (TEAM) Initiative. The Center for Applied Biodiversity Science (CABS). Conservación Internacional. Watkinsville, EE. UU

Schultz, P. G. 2003. Structure and Diversity of the Forests at the El Eden Ecological Reserve. Pp. 91-114, in The Lowland Maya Area: three millennia at the human-wildland interface. The Haworth Press. New York. EE.UU.

SILVER, S. 2004. Estimando la abundancia de jaguares mediante trampas-cámara. Wildlife Conservation Society. New York, EE. UU. Solsalo, M. K., y M. C. Cavalcanti. 2006. Estimating the density of jaguar population in the Brazilian Pantanal using cameratraps and capture-recapture sampling in combination with GPS radio telemetry. Biological Conservation 129:487-496.

Sunquist, M., y F. Sunquist. 2002. Wild Cats of the world, The University of Chicago Press. Chicago, EE.UU.

Sunquist, M. 1991. The ecology of ocelot: the importance of incorporating life history traits into conservation plans. Pp.117-128 in Felinos de Venezuela: Biología, Ecología y Conservación. Fudeci, Venezuela.

Valdez-Jiménez, D., C. M. García-Balderas, y G. E. Quintero-Díaz. 2013. Presencia del ocelote (Leopardus pardalis) en la "Sierra del Laurel", municipio de Calvillo, Aguascalientes, México. Acta Zoológica Mexicana (n. s.) 29:688-692.

Zaragoza-Quintana, E. P., J. M. Pech-Canché., J. E. Sosa-Escalante., S. Hernández-Betancourt, L. S. León-Paniagua, y M. C. MacSwiney. 2016. Small rodents in the Yucatan Peninsula: knowledge and perspectives in 114 years of research. Therya 7:299-314.

Associated editor: Cristina Macswiney

Submitted: August 22, 2016; Reviewed: September 30, 2016;

Accepted: November 7, 2016; Published on line January 13, 2017. 